\title{
Estimated Annual Numbers of Foodborne Pathogen-Associated Illnesses, Hospitalizations, and Deaths, France, 2008-2013
}

\author{
Dieter Van Cauteren, Yann Le Strat, Cécile Sommen, Mathias Bruyand, Mathieu Tourdjman, \\ Nathalie Jourdan-Da Silva, Elisabeth Couturier, Nelly Fournet, Henriette de Valk, Jean-Claude Desenclos
}

\begin{abstract}
Estimates of the annual numbers of foodborne illnesses and associated hospitalizations and deaths are needed to set priorities for surveillance, prevention, and control strategies. The objective of this study was to determine such estimates for 2008-2013 in France. We considered 15 major foodborne pathogens (10 bacteria, 3 viruses, and 2 parasites) and estimated that each year, the pathogens accounted for 1.28-2.23 million illnesses, 16,500-20,800 hospitalizations, and 250 deaths. Campylobacter spp., nontyphoidal Salmonella spp. and norovirus accounted for $>70 \%$ of all foodborne pathogen-associated illnesses and hospitalizations; nontyphoidal Salmonella spp. and Listeria monocytogenes were the main causes of foodborne pathogen-associated deaths; and hepatitis $\mathrm{E}$ virus appeared to be a previously unrecognized foodborne pathogen causing $\approx 68,000$ illnesses in France every year. The substantial annual numbers of foodborne illnesses and associated hospitalizations and deaths in France highlight the need for food-safety policymakers to prioritize foodborne disease prevention and control strategies.
\end{abstract}

Goodborne pathogens are of public health concern - worldwide (1). Estimates of the total number of foodborne illnesses and associated hospitalizations and deaths are needed to assess their effect on health and to set priorities for surveillance, prevention, and control strategies. In 2000, the number of foodborne illnesses and associated deaths in France was estimated by using data from 1990 2000. However, for most pathogens, data were lacking to derive estimates at the population level (2).

Since that study, specific surveillance systems have been implemented in France for Campylobacter spp. (2002) (3), hepatitis A virus (2005), and hepatitis E virus (2002) (4). Additional surveys have been conducted to provide information on healthcare-seeking behavior and the incidence of acute gastroenteritis in the general population (2009-2010) (5) and on physician practices in requesting

Author affiliation: Santé Publique France, Saint-Maurice, France

DOI: https://doi.org/10.3201/eid2309.170081 fecal samples for patients with acute gastroenteritis (20132014) (6). Furthermore, the quality and availability of other nonspecific data sources (e.g., hospital discharge registers and health insurance reimbursement data) have improved and are increasingly used for epidemiologic studies in France (7-9). Thus, recent and valid data are available to estimate the population-level health effects of several foodborne pathogens. Such estimates have recently been generated for Campylobacter spp. and nontyphoidal Salmonella spp. (hereafter referred to as Salmonella spp.), the 2 main causes of foodborne bacterial infections in France (10). Taking into account this improved knowledge and data availability, we conducted a study to estimate the annual number of illnesses, hospitalizations, and deaths associated with 15 foodborne pathogens in France.

\section{Methods}

Using data sources from 2008-2013, we estimated the number of illnesses, hospitalizations, and deaths in France resulting from 15 foodborne pathogens: 10 bacteria ( $\mathrm{Ba}$ cillus cereus, Campylobacter spp., Clostridium botulinum, Clostridium perfringens, Shiga-toxin-producing Escherichia coli [STEC], Listeria monocytogenes, Salmonella spp., Shigella spp., Staphylococcus aureus, Yersinia spp.); 3 viruses (hepatitis A virus, hepatitis E virus, norovirus); and 2 parasites (Taenia saginata, Toxoplasma gondii). We used France's 2010 census population $(62,765,235$ persons) for the estimates.

We used different statistical models, depending on the most suitable data available for each pathogen, with many inputs to estimate the number of illnesses, hospitalizations, and deaths (online Technical Appendix Table 1, https:// wwwnc.cdc.gov/EID/article/23/9/17-0081-Techapp1.pdf). For most proportions we defined a lower and upper bound and a beta distribution with 2 parameters derived from a method of moments, assuming a mean $m=($ lower + upper bound) $/ 2$ and an $\mathrm{SD}=($ upper bound $-m) / 2(11)$. We used lognormal probability distributions for model inputs derived from a national survey on acute gastroenteritis in 
France (5) and for the annual numbers of reported illnesses, hospitalizations, and deaths. For final estimates, we multiplied the distributions by using Monte Carlo simulation (10,000 iterations) with $\mathrm{R}$ version 3.3.2 (12). We report median values and use ranges between the 5th and 95th percentiles of the output distribution to define a $90 \%$ credible interval $\left(\mathrm{CrI}_{90 \%}\right)$.

\section{IIInesses}

To estimate the numbers of illnesses, we obtained surveillance data from the mandatory notification system (C. botulinum, L. monocytogenes, hepatitis A virus, and foodborne disease outbreaks) and from national reference laboratories and their laboratory surveillance networks (C. botulinum, Campylobacter spp., STEC, L. monocytogenes, Salmonella spp., Shigella spp., Yersinia spp., hepatitis A virus, hepatitis $\mathrm{E}$ virus, and $T$. gondii). Inclusion in these surveillance systems implies that the ill person sought medical care, had laboratory testing prescribed, and had a specimen submitted for laboratory testing and that the laboratory identified the pathogen and reported the positive result to the surveillance system. These steps can be summarized into 2 multiplication factors: an underreporting factor defined as the match between the total number of laboratory-confirmed illnesses and the number of laboratory-confirmed illnesses reported to the surveillance system; and an underdiagnosis factor taking into account the proportion of cases that were not laboratory-confirmed because the patient did not seek medical advice or was misdiagnosed. We took both multiplication factors into account to estimate the number of illnesses from mandatory notification data and national reference laboratory data.

Previously published parameters for estimating the number of Campylobacter spp.- and Salmonella spp.-associated illnesses $(10)$ were used as a proxy to estimate the level of underdiagnosis for Yersinia spp. (using Campylobacter spp. data) and Shigella spp. (using Salmonella spp. data). For C. botulinum and L. monocytogenes, we assumed that $80 \%-100 \%$ of the cases were in persons who sought medical care and had laboratory-confirmed diagnoses. To account for underreporting, we conducted ad hoc laboratory surveys for Campylobacter spp., Salmonella spp., Shigella spp., and Yersinia spp., and we conducted a capturerecapture study for $L$. monocytogenes.

In France, cases of B. cereus, S. aureus, and C. perfringens infection are notified only through mandatory notification of point-source foodborne disease outbreaks. For these pathogens, we assumed that the multiplier between the number of confirmed outbreak cases and the number of community cases of foodborne origin would be similar to that estimated for Salmonella spp. We estimated the number of illness caused by $T$. gondii and hepatitis A and E viruses from seroprevalence data and the number of illnesses caused by $T$. saginata from health insurance reimbursement data for niclosamide (a drug used to treat tapeworm infestation). We used data from the literature to estimate the number of illnesses caused by STEC. To estimate the number of norovirus cases, we applied a proportion (14\%$22 \%$ ) of norovirus-associated acute gastroenteritis cases to the annual number of acute gastroenteritis illnesses in France (Table 1). This proportion was based on findings from a 2008-2009 community study in the United Kingdom (13) and a meta-analysis of 175 studies published during 1990-2014 (14). Model inputs used for each pathogen are presented in online Technical Appendix Table 1.

\section{Hospitalizations}

We used the French Hospital Information System (FHIS) as the main data source for estimating the number of hospitalizations. The system is a national database of hospital records that contains sociodemographic information (age, sex, and residence area) and medical information (main cause for admission, concurrent medical conditions, modes of admission, and discharge) (10). Diseases are coded according to the International Classification of Diseases, 10th revision (ICD-10; http://www.who.int/classifications/icd/ en/). We extracted all hospital records with a patient discharge date during January 2008-December 2013 and containing an ICD-10 code of interest as the main cause for admission or as a concurrent medical condition.

We used the number of hospital records with pathogen-specific ICD-10 codes to estimate the annual number of hospitalizations for 8 pathogens, 4 of which cause acute gastroenteritis (Table 2). We did not redistribute records with only unspecified gastroenteritis codes to the 8 pathogens, but we did correct for undercapture, taking into account the proportion of fecal samples tested for each pathogen and the sensitivity of fecal culture. When data were available, we compared trends over time and patient age

\begin{tabular}{lc}
\hline \multicolumn{2}{l}{ Table 1. Data sources used to estimate the number of pathogen- } \\
specific illnesses, France, 2008-2013 & \\
\hline Pathogen & Data source \\
\hline Bacillus cereus & Surveillance \\
Campylobacter spp. & Surveillance \\
Clostridium botulinum & Surveillance \\
Clostridium perfringens & Surveillance \\
Hepatitis A virus & Seroprevalence \\
Hepatitis E virus & Seroprevalence \\
Listeria monocytogenes & Surveillance \\
Norovirus & Literature and national \\
& telephone survey \\
Salmonella spp., nontyphoidal & Surveillance \\
Shiga toxin-producing Escherichia coli & Literature \\
Shigella spp. & Surveillance \\
Staphylococcus aureus & Surveillance \\
Taenia saginata & Health insurance \\
& reimbursement data \\
Toxoplasma gondii & Seroprevalence \\
Yersinia spp. & Surveillance \\
\hline
\end{tabular}


Table 2. Methods used to estimate the number of pathogen-specific hospitalizations, France, 2008-2013*

\begin{tabular}{|c|c|}
\hline Pathogen & Method \\
\hline Bacillus cereus & Proportion of hospitalizations for AG applied to annual no. of illnesses for the pathogen \\
\hline Campylobacter spp. & Annual no. persons hospitalized with a specific ICD-10 code in FHIS \\
\hline Clostridium botulinum & Mandatory notification data \\
\hline Clostridium perfringens & Proportion of hospitalizations for AG applied to annual no. of illnesses for the pathogen \\
\hline Hepatitis A virus & Annual no. persons hospitalized with a specific ICD-10 code in FHIS \\
\hline Hepatitis E virus & Annual no. persons hospitalized with a specific ICD-10 code in FHIS \\
\hline Listeria monocytogenes & Mandatory notification data \\
\hline Norovirus & Proportion of hospitalizations for $A G$ applied to annual no. of illnesses for the pathogen \\
\hline Salmonella spp., nontyphoidal & Annual no. persons hospitalized with a specific ICD-10 code in FHIS \\
\hline Shiga toxin-producing Escherichia coli & Salmonella spp. and Campylobacter spp. data used as a proxy \\
\hline Shigella spp. & Annual no. persons hospitalized with a specific ICD-10 code in FHIS \\
\hline Staphylococcus aureus & Proportion of hospitalizations for AG applied to annual no. of illnesses for the pathogen \\
\hline Taenia saginata & Annual no. persons hospitalized with a specific ICD-10 code in FHIS \\
\hline Toxoplasma gondii & Annual no. persons hospitalized with a specific ICD-10 code in FHIS \\
\hline Yersinia spp. & Annual no. persons hospitalized with a specific ICD-10 code in FHIS \\
\hline
\end{tabular}

and sex distributions of the hospital data with surveillance data from the national reference laboratories (Campylobacter spp., Salmonella spp., Shigella spp., Yersinia spp., and hepatitis $\mathrm{E}$ virus) and with mandatory notification data (hepatitis A virus).

We used the number of hospital records with acute gastroenteritis-associated ICD-10 codes (A00-A06.2 and A06.9-A09.9) to estimate the annual number of persons hospitalized for acute gastroenteritis. We then divided that number by the total number of persons with acute gastroenteritis to estimate the percentage of those persons who were hospitalized $(0.58 \%-0.75 \%)$ (online Technical Appendix Table 1). For norovirus, B. cereus, C. perfringens, and $S$. aureus, we applied the proportion of hospitalizations for acute gastroenteritis to the annual number of illnesses for each pathogen to estimate the annual number of hospitalizations. For STEC, we used the proportion of hospitalizations estimated for Salmonella spp. and Campylobacter spp. as a proxy. For C. botulinum and L. monocytogenes, we used surveillance data from the mandatory notification system (Table 2 ).

\section{Deaths}

We explored death certificate data from the French national mortality database (Institut National de la Santé et de la Recherche Médicale, CépiDc [Epidemiology Center on Medical Causes of Death]) and data from FHIS to estimate the number of foodborne illness-associated deaths. For both data sources, we extracted all records for 2008-2013 with an ICD-10 code of interest as the main cause of death or hospitalization or as a concurrent medical condition. Compared with data from FHIS, death certificates contained fewer pathogen-specific ICD-10 codes; therefore, we used the hospital information system data as the main data source for estimating the number of deaths.

To estimate the number of deaths from Campylobacter spp., Salmonella spp., Shigella spp., Yersinia spp., hepatitis
A and $\mathrm{E}$ viruses, $T$. saginata, and $T$. gondii infections, we used the number of hospital records with a pathogen-specific ICD-10 code and death shown as the mode of discharge. To estimate the number of norovirus-associated deaths, we applied the proportion of deaths among hospitalized casepatients with an ICD-10 code associated with viral gastroenteritis (ICD-10 codes A08.0-A08.4) to the annual number of hospitalizations for norovirus $(0.18 \%-0.30 \%$; online Technical Appendix Table 1). This proportion was also used as a proxy to estimate the number of deaths from B. cereus-, $C$. perfringens-, and S. aureus-associated hospitalizations. For C. botulinum and L. monocytogenes, we used mandatory notification data to estimate the number of deaths (Table 3 ).

\section{Foodborne Transmission}

To estimate the number of foodborne illnesses and associated hospitalizations and deaths, we applied a pathogenspecific proportion of foodborne transmission (online Technical Appendix Table 2). For 11 of the 15 pathogens, we used estimates published in the United States in 2011 (15). For norovirus and hepatitis A virus, data from more recent studies were used (16,17). For hepatitis E virus and $T$. saginata, the proportions of foodborne transmission were estimated on the basis of discussions with experts from the French Public Health Agency.

\section{Results}

Overall, the pathogens included in our study accounted for 4.9 million cases of illness ( $\mathrm{CrI}_{90 \%} 4.2-6.2$ million), 42,500 hospitalizations $\left(\mathrm{CrI}_{90 \%} 37,242-50,526\right)$, and 368 deaths $\left(\mathrm{CrI}_{90 \%} 335-486\right)$ each year in France. Of those 4.9 million cases, 1.5 million were caused by foodborne pathogens ( $\mathrm{CrI}_{90 \%}$ 1.28-2.23 million), of which 880,500 (59\%) were caused by bacteria; 579,500 (38\%) by viruses; and 45,000 (3\%) by parasites. These foodborne illnesses led to 17,281 hospitalizations $\left(\mathrm{CrI}_{90 \%}, 15,520-20,785\right)$ and 248 deaths $\left(\mathrm{CrI}_{90 \%} 223-350\right)$. 
Table 3. Methods used to estimate the number of pathogen-specific deaths, France, 2008-2013*

\begin{tabular}{lc}
\hline Pathogen & Method \\
\hline Bacillus cereus & Hospital discharge data with viral gastroenteritis-associated ICD-10 codes \\
Campylobacter spp. & Hospital discharge data with pathogen-specific ICD-10 codes \\
Clostridium botulinum & Mandatory notification data \\
Clostridium perfringens & Hospital discharge data with viral gastroenteritis-associated ICD-10 codes \\
Hepatitis A virus & Hospital discharge data with pathogen-specific ICD-10 codes \\
Hepatitis E virus & Hospital discharge data with pathogen-specific ICD-10 codes \\
Listeria monocytogenes & Mandatory notification data \\
Norovirus & Hospital discharge data with viral gastroenteritis-associated ICD-10 codes \\
Salmonella spp., nontyphoidal & Hospital discharge data with pathogen-specific ICD-10 codes \\
Shiga toxin-producing Escherichia coli & Salmonella spp. and Campylobacter spp. data used as a proxy \\
Shigella spp. & Hospital discharge data with pathogen-specific ICD-10 codes \\
Staphylococcus aureus & Hospital discharge data with viral gastroenteritis-associated ICD-10 codes \\
Taenia saginata & Hospital discharge data with pathogen-specific ICD-10 codes \\
Toxoplasma gondii & Hospital discharge data with pathogen-specific ICD-10 codes \\
Yersinia spp. & Hospital discharge data with pathogen-specific ICD-10 codes \\
\hline${ }^{*}$ ICD-10, International Classification of Diseases, 10th Revision (http://www.who.int/classifications/icd/en/). \\
\hline \multicolumn{2}{l}{. }
\end{tabular}

Norovirus ranked first as the cause of foodborne illnesses $(34 \%)$, third as a cause for foodborne illness-associated hospitalizations $(20 \%)$, and seventh as a cause of foodborne illness-associated deaths (3\%). Salmonella spp. ranked third as the cause of foodborne illnesses $(12 \%)$, second as a cause for hospitalization (24\%), and first as a cause of death (27\%). $L$ monocytogenes ranked second (26\%), before Campylobacter spp. (17\%), as a cause of foodborne illness-associated deaths (online Technical Appendix Table 2).

\section{Discussion}

We estimated the population-level number of illnesses, hospitalizations, and deaths in France caused by 15 pathogens with the potential for foodborne transmission. Campylobacter spp., Salmonella spp., and norovirus were responsible for $73 \%$ of all foodborne illnesses and $76 \%$ of all associated hospitalizations. The pathogens that cause most foodborne illnesses or hospitalizations are not necessarily those that cause the most deaths: L. monocytogenes caused $<0.1 \%$ of all foodborne illnesses but ranked second as a cause of foodborne illness-associated deaths, just behind Salmonella spp.

We used different approaches, depending on the most suitable data that were available, to generate estimates. We could not easily compare our results with previous estimates from France (2) and other countries because of different data sources, assumptions, and methods. Nevertheless, recent estimates of the burden of foodborne illnesses in the European region also indicated that the 3 most frequent causes of foodborne illness were norovirus (ranked first), Campylobacter spp. (second), and Salmonella spp. (third) (1). These pathogens were also among the leading causes of foodborne illnesses and hospitalizations in North America $(15,18)$ and Oceania $(19,20)$. Salmonella spp. and L. monocytogenes accounted for $\approx 50 \%$ of all foodborne illness-associated deaths in France, and were also responsible for most foodborne illness-associated deaths in other high-income countries $(1,15,18-20)$.
We estimated the number of most pathogen-specific illnesses by using laboratory-based surveillance data corrected for underreporting and underdiagnosis, and we used well-documented estimates for Campylobacter spp. and Salmonella spp. (10). We assumed that the parameters regarding healthcare-seeking behavior and laboratory practice for Yersinia spp. and Shigella spp. were similar to those for Campylobacter spp. and Salmonella spp., respectively. The validity of these assumptions is difficult to explore; further studies would be needed to produce more robust estimates of the true level of underdiagnosis for these 2 pathogens in France.

For $B$. cereus, $C$. perfringens, and $S$. aureus, we assumed that the multiplier between the number of outbreak cases and the number of foodborne illnesses would be similar to that for Salmonella spp. An alternative approach for $C$. perfringens would have been to apply a proportion of acute gastroenteritis cases by this pathogen estimated in the United Kingdom $(0.3-1.7 \%)(13)$ to the annual number of acute gastroenteritis illnesses in France. This approach would result in an estimate $\left(\mathrm{CrI}_{90 \%} 84,450-278,964\right)$ within the range of the estimate in our study. The estimates for B. cereus, C. perfringens, and $S$. aureus indicate that the effect of these pathogens in terms of foodborne illnesses appears to be high in France. However, only foodborne illness outbreak data were available to estimate the number of illnesses for these pathogens, and more data are needed to confirm our estimates.

We included hepatitis $E$ virus in our study because, in France, indigenous cases of hepatitis $\mathrm{E}$ have been shown to be associated with foodborne transmission, particularly through consumption of products containing undercooked or raw pork liver $(21,22)$. We estimated the number of hepatitis E cases in France from a seroprevalence study conducted in 2013, and the proportion of cases caused by foodborne transmission was assumed to be between $75 \%$ and $100 \%$. Further studies, in particular on the proportion of foodborne transmission of hepatitis E in France, are needed to confirm these estimates. 
Our use of seroprevalence and health insurance drug reimbursement data to estimate the numbers of $T$. gondiiand $T$. saginata-associated foodborne illnesses was similar to methods previously used in France (2). Our results indicated a decrease in the number of foodborne illnesses over the past decade (from 51,600 to 12,000 cases for $T$. gondii and from 64,500 to 33,000 cases for T. saginata). These decreases may be explained by fewer exposures to the parasites (23), by changes in food habits, and by improved hygiene practices in meat production. For $T$. saginata, the number of illnesses may be underestimated because the decrease might also be explained by a shift of treatment from niclosamide to praziquantel for this infection over the past decade in France.

We estimated the number of illnesses caused by norovirus by applying a proportion of acute gastroenteritis cases caused by this pathogen to the annual number of acute gastroenteritis illnesses in France. The final estimate for France is lower than that for other countries that used a similar method $(15,18)$, primarily because of a lower estimated incidence of acute gastroenteritis in France (5) but also because we used a lower proportion of foodborne norovirus transmission $(12 \%-16 \%)$ on the basis of an extensive study published in 2015 (16). Despite these differences and their effect on the final estimate, norovirus ranked first in terms of foodborne illnesses in France and appears to be a key foodborne cause of acute gastroenteritis.

The FHIS was our main data source for estimating numbers of hospitalizations and deaths associated with the 15 pathogens in our study. The relevance of this data source may be questioned because of limitations in diagnosis accuracy and in consistency of disease coding. For most of the pathogens, we estimated the number of hospitalizations by using the number of hospital records with specific ICD-10 codes. We compared trends over time and age and sex distributions of the hospital data with surveillance data from the national reference laboratories and with mandatory notification data. Trends and distributions were similar between the different data sources, supporting the use of FHIS data to estimate the number of hospitalizations. For Campylobacter spp., Salmonella spp., Yersinia spp., and Shigella spp., we corrected the number of hospitalizations and deaths for underdiagnosis, taking into account a proportion of fecal samples tested for each pathogen and the sensitivity of fecal culture. However, for the other pathogens, no specific underdiagnosis multiplier could be estimated and, therefore, the estimates presented in this study are probably conservative. An overestimation is also possible if the pathogen of interest did not cause the illness that led to the hospitalization but was, nevertheless, coded as a concurrent medical condition.

A high number of hospitalizations due to acute gastroenteritis were reported in the FHIS without a specific
ICD-10 code because not all hospitalized patients were systematically tested for all pathogens that cause acute gastroenteritis. We used the proportion of hospitalizations for acute gastroenteritis as a proxy to estimate the number of hospitalizations for norovirus, B. cereus, C. perfringens, and $S$. aureus because testing for these pathogens is infrequently performed in France and because these pathogens cause illnesses with similar symptoms and severity. This proportion $(0.58 \%-0.75 \%)$ is lower than that estimated for Campylobacter spp. (0.9\%-1.9\%) and for Salmonella spp. $(1.2 \%-3.6 \%)$, which is plausible considering that illness caused by B. cereus, C. perfringens, and $S$. aureus is less severe than that caused by Campylobacter spp. and Salmonella spp. Data sources described in the literature to estimate the number of hospitalizations for norovirus, $B$. cereus, C. perfringens, and $S$. aureus infections include hospital discharge data and data from foodborne disease outbreaks $(15,18,19,24,25)$. Estimating the number of hospitalizations for these pathogens is challenging, and these different methodologic approaches have a major effect on the final estimate. For norovirus, despite differences in methodology and healthcare systems, our estimate (all modes of transmission) of the number of hospitalizations was in the same range as those estimated in North America $(24,25)$ and in the Netherlands (26).

Data to estimate the number of deaths associated with foodborne illnesses are scarce and difficult to obtain. We explored death certificate data but decided not to use that source because few records contained pathogen-specific ICD-10 codes. Hospital discharge data were the only or the most reliable data source available to estimate the number of deaths for most pathogens included in this study. However, deaths may occur after hospitalization discharge or without hospitalization at all. Therefore, our estimates are uncertain and are probably underestimated, even though we did not take into account the possibility that underlying concurrent conditions, not foodborne pathogens, may have caused or contributed to death.

As pointed out in the literature, difficulties in accurately determining the proportion of foodborne pathogen transmission is a key factor contributing to the uncertainty of foodborne illness estimates $(15,27)$. Different methodologic approaches, such as epidemiologic and microbiologic approaches, intervention studies, and expert elicitation, have been used to estimate the proportion of foodborne transmission $(15,28-32)$. Overall, in high-income countries, foodborne transmission has been considered a major transmission route for several bacterial pathogens (B. cereus, Campylobacter spp., C. perfringens, L. monocytogenes, Salmonella spp., S. aureus) and a minor transmission route for norovirus and hepatitis A virus. Nevertheless, comparison of the estimates by using expert elicitation shows greater variability and higher uncertainties, depending on 
how the experts were recruited, the expert panel size, or the elicitation method used $(27,33)$. We decided to use the proportion of foodborne transmission published in the United States in 2011 (15) as these proportions were based on epidemiologic and microbiologic data rather than expert elicitation. It is possible that food consumption patterns and frequency and type of microbiologic contamination differ between the United States and France and may influence pathogen exposure, resulting in a different proportion of foodborne pathogen transmission in the 2 countries. Further research is needed to obtain specific source attribution estimates for France.

The 15 foodborne pathogens in our study were selected on the basis of their perceived public health significance, their occurrence in France, and the availability of a minimum of data. Other known pathogens with potential foodborne transmission exist (e.g., other non-STEC pathogenic E.coli, rotavirus, and Cryptosporidium spp.), and the total numbers of foodborne illnesses and associated hospitalizations and deaths presented in this study are likely conservative.

We took into account new data sources that allowed for accurate estimates of foodborne illnesses and associated hospitalizations and deaths at the community level in France. Our estimates entail several assumptions, and a high degree of uncertainty remains for some of them. Our estimates indicate that substantial numbers of foodborne pathogen-associated illnesses, hospitalizations, and deaths occur each year in France, necessitating the prioritization of prevention and control strategies by food safety policymakers. We did not specifically consider the effect of sequelae linked to these illnesses when generating our estimates. Thus, our findings capture only part of the overall effect of foodborne infections, and they clear the way for further research on the public health burden of foodborne pathogens in France, taking into account complications and sequelae.

\section{Acknowledgments}

We thank Javier Nicolau, Marjorie Boussac, Laure Fonteneau, Francis Megraud, François-Xavier Weill, Simon Le Hello, Thierry Blanchon, Clement Turbelin, and Véronique Vaillant for providing data and expert advice for these estimates.

Dr. Van Cauteren is epidemiologist at the Department of Infectious Diseases at Santé Publique France, the French National Public Health Agency. His research focuses on surveillance and the burden of foodborne infections.

\section{References}

1. Kirk MD, Pires SM, Black RE, Caipo M, Crump JA, Devleesschauwer B, et al. World Health Organization estimates of the global and regional disease burden of 22 foodborne bacterial, protozoal, and viral diseases, 2010: a data synthesis. PLoS Med. 2015;12:e1001921. http://dx.doi.org/ 10.1371/journal.pmed.1001921
2. Vaillant V, de Valk H, Baron E, Ancelle T, Colin P, Delmas MC, et al. Foodborne infections in France. Foodborne Pathog Dis. 2005;2:221-32. http://dx.doi.org/10.1089/fpd.2005.2.221

3. Gallay A, Simon F, Mégraud F. Surveillance of human Campylobacter infections in France-part 2-implementation of national surveillance. Euro Surveill. 2003;8:218.

4. Couturier E. Hepatitis E: synthesis in human epidemiology [in French]. Bulletin épidémiologique hebdomadaire, 2010; Special issue: $18-9$

5. Van Cauteren D, De Valk H, Vaux S, Le Strat Y, Vaillant V. Burden of acute gastroenteritis and healthcare-seeking behaviour in France: a population-based study. Epidemiol Infect. 2012;140:697705. http://dx.doi.org/10.1017/S0950268811000999

6. Van Cauteren D, Turbelin C, Fonteneau L, Hanslik T, De Valk H, Blanchon T. Physician practices in requesting stool samples for patients with acute gastroenteritis, France, August 2013-July 2014. Epidemiol Infect. 2015;143:2532-8. http://dx.doi.org/10.1017/ S0950268814003884

7. Gabet A, Juillière Y, Lamarche-Vadel A, Vernay M, Olié V. National trends in rate of patients hospitalized for heart failure and heart failure mortality in France, 2000-2012. Eur J Heart Fail. 2015;17:583-90. http://dx.doi.org/10.1002/ejhf.284

8. Tuppin P, Rivière S, Rigault A, Tala S, Drouin J, Pestel L, et al. Prevalence and economic burden of cardiovascular diseases in France in 2013 according to the national health insurance scheme database. Arch Cardiovasc Dis. 2016;109:399-411. http://dx.doi.org/10.1016/j.acvd.2016.01.011

9. van Cauteren D, Millon L, de Valk H, Grenouillet F. Retrospective study of human cystic echinococcosis over the past decade in France, using a nationwide hospital medical information database. Parasitol Res. 2016;115:4261-5. http://dx.doi.org/10.1007/ s00436-016-5204-1

10. Van Cauteren D, De Valk H, Sommen C, King LA, Jourdan-Da Silva N, Weill FX, et al. Community incidence of campylobacteriosis and nontyphoidal salmonellosis, France, 2008-2013. Foodborne Pathog Dis. 2015;12:664-9. http://dx.doi.org/10.1089/fpd.2015.1964

11. Spiegelhalter DJ, Abrams KR, Myles JP. Bayesian approaches to clinical trials and health-care evaluation. Chichester (UK): Wiley; 2004.

12. R Development Core Team. R: a language and environment for statistical computing. Vienna: R Foundation for Statistical Computing; 2016.

13. Tam CC, O'Brien SJ, Tompkins DS, Bolton FJ, Berry L, Dodds J, et al.; IID2 Study Executive Committee. Changes in causes of acute gastroenteritis in the United Kingdom over 15 years: microbiologic findings from 2 prospective, population-based studies of infectious intestinal disease. Clin Infect Dis. 2012; 54:1275-86. http://dx.doi.org/10.1093/cid/cis028

14. Ahmed SM, Hall AJ, Robinson AE, Verhoef L, Premkumar P, Parashar UD, et al. Global prevalence of norovirus in cases of gastroenteritis: a systematic review and meta-analysis. Lancet Infect Dis. 2014;14:725-30. http://dx.doi.org/10.1016/ S1473-3099(14)70767-4

15. Scallan E, Hoekstra RM, Angulo FJ, Tauxe RV, Widdowson MA, Roy SL, et al. Foodborne illness acquired in the United Statesmajor pathogens. Emerg Infect Dis. 2011;17:7-15. http://dx.doi.org/10.3201/eid1701.P11101

16. Verhoef L, Hewitt J, Barclay L, Ahmed SM, Lake R, Hall AJ, et al. Norovirus genotype profiles associated with foodborne transmission, 1999-2012. Emerg Infect Dis. 2015;21:592-9. http://dx.doi.org/10.3201/eid2104.141073

17. Petrignani M, Verhoef L, Vennema H, van Hunen R, Baas D, van Steenbergen JE, et al. Underdiagnosis of foodborne hepatitis A, the Netherlands, 2008-2010. Emerg Infect Dis. 2014;20:596-602. http://dx.doi.org/10.3201/eid2004.130753 
18. Thomas MK, Murray R, Flockhart L, Pintar K, Fazil A, Nesbitt A, et al. Estimates of foodborne illness-related hospitalizations and deaths in Canada for 30 specified pathogens and unspecified agents. Foodborne Pathog Dis. 2015;12:820-7. http://dx.doi.org/10.1089/ fpd.2015.1966

19. Kirk M, Ford L, Glass K, Hall G. Foodborne illness, Australia, circa 2000 and circa 2010. Emerg Infect Dis. 2014;20:1857-64. http://dx.doi.org/10.3201/eid2011.131315

20. Lake RJ, Cressey PJ, Campbell DM, Oakley E. Risk ranking for foodborne microbial hazards in New Zealand: burden of disease estimates. Risk Anal. 2010;30:743-52. http://dx.doi.org/10.1111/ j.1539-6924.2009.01269.x

21. Guillois Y, Abravanel F, Miura T, Pavio N, Vaillant V, Lhomme S, et al. high proportion of asymptomatic infections in an outbreak of hepatitis E associated with a spit-roasted piglet, France, 2013. Clin Infect Dis. 2016;62:351-7. http://dx.doi.org/10.1093/cid/civ862

22. Renou C, Roque-Afonso AM, Pavio N. Foodborne transmission of hepatitis E virus from raw pork liver sausage, France. Emerg Infect Dis. 2014;20:1945-7. http://dx.doi.org/10.3201/eid2011.140791

23. Nogareda F, Le Strat Y, Villena I, De Valk H, Goulet V. Incidence and prevalence of Toxoplasma gondii infection in women in France, 1980-2020: model-based estimation. Epidemiol Infect. 2014;142:1661-70. http://dx.doi.org/10.1017/S0950268813002756

24. Lopman BA, Hall AJ, Curns AT, Parashar UD. Increasing rates of gastroenteritis hospital discharges in US adults and the contribution of norovirus, 1996-2007. Clin Infect Dis. 2011;52:466-74. http://dx.doi.org/10.1093/cid/ciq163

25. Morton VK, Thomas MK, McEwen SA. Estimated hospitalizations attributed to norovirus and rotavirus infection in Canada, 2006-2010. Epidemiol Infect. 2015;143:3528-37. http://dx.doi.org/ $10.1017 / \mathrm{S} 0950268815000734$

26. Havelaar AH, Haagsma JA, Mangen MJ, Kemmeren JM, Verhoef LP, Vijgen SM, et al. Disease burden of foodborne pathogens in the Netherlands, 2009. Int J Food Microbiol. 2012; 156:231-8. http://dx.doi.org/10.1016/j.ijfoodmicro. 2012.03.029
27. Glass K, Ford L, Kirk MD. Drivers of uncertainty in estimates of foodborne gastroenteritis incidence. Foodborne Pathog Dis. 2014;11:938-44. http://dx.doi.org/10.1089/fpd.2014.1816

28. Hald T, Aspinall W, Devleesschauwer B, Cooke R, Corrigan T, Havelaar AH, et al. World Health Organization estimates of the relative contributions of food to the burden of disease due to selected foodborne hazards: a structured expert elicitation. PLoS One. 2016;11:e0145839. http://dx.doi.org/10.1371/journal.pone.0145839

29. Batz MB, Hoffmann S, Morris JG Jr. Ranking the disease burden of 14 pathogens in food sources in the United States using attribution data from outbreak investigations and expert elicitation. J Food Prot. 2012;75:1278-91. http://dx.doi.org/10.4315/ 0362-028X.JFP-11-418

30. Butler AJ, Thomas MK, Pintar KD. Expert elicitation as a means to attribute 28 enteric pathogens to foodborne, waterborne, animal contact, and person-to-person transmission routes in Canada. Foodborne Pathog Dis. 2015;12:335-44. http://dx.doi.org/10.1089/ fpd.2014.1856

31. Havelaar AH, Galindo AV, Kurowicka D, Cooke RM. Attribution of foodborne pathogens using structured expert elicitation. Foodborne Pathog Dis. 2008;5:649-59. http://dx.doi.org/10.1089/ fpd.2008.0115

32. Vally H, Glass K, Ford L, Hall G, Kirk MD, Shadbolt C, et al. Proportion of illness acquired by foodborne transmission for nine enteric pathogens in Australia: an expert elicitation. Foodborne Pathog Dis. 2014;11:727-33. http://dx.doi.org/10.1089/fpd.2014.1746

33. Butler AJ, Thomas MK, Pintar KD. Systematic review of expert elicitation methods as a tool for source attribution of enteric illness. Foodborne Pathog Dis. 2015;12:367-82. http://dx.doi.org/10.1089/ fpd.2014.1844

Address for correspondence: Dieter Van Cauteren, Department of Infectious Diseases, Santé Publique France, French National Public Health Agency, F-94415, 12 rue du Val d'Osne, Saint-Maurice,

France; email: vancauteren.dieter@hotmail.com

\section{EID Adds Advanced Search Features for Articles}

Emerging Infectious Diseases now has an advanced search feature that makes it easier to find articles by using keywords, names of authors, and specified date ranges. You can sort and refine search results by manuscript number, volume or issue number, or article type. A quick start guide and expandable help section show you how to optimize your searches.

https://wwwnc.cdc.gov/eid/AdvancedSearch

EID's new mapping feature allows you to search for articles from specific countries by using a map or table to locate countries. You can refine search results by article type, volume and issue, and date, and bookmark your search results.

https://wwwnc.cdc.gov/eid/ArticleMap 